\title{
Leveraging risk communication science across US federal agencies
}

\author{
Many US federal agencies apply principles from risk communication science across a wide variety of hazards. \\ In so doing, they identify key research and practice gaps that, if addressed, could help better serve the nation's \\ communities and greatly enhance practice, research, and policy development.
}

\author{
William M. P. Klein, Alycia K. Boutté, Heather Brake, Madeline Beal, Katherine Lyon-Daniel, \\ Emily Eisenhauer, Monica Grasso, Bryan Hubbell, Karen E. Jenni, Christopher J. Lauer, Arthur W. Lupia, \\ Christine E. Prue, Paula Rausch, Carl D. Shapiro, Michael D. Smith and William T. Riley
}

T he SARS-CoV-2 pandemic has brought into sharp relief the importance and complexities of effective risk communication. Risk and uncertainty are inherent in many contexts, including natural disasters, regional and international conflicts, and pandemics. A prominent challenge for risk communicators in these contexts is that people tend not to construe risk as statisticians do, focusing on emotional and social factors more than quantitative information ${ }^{1}$. Moreover, access and receptiveness to risk communications are greatly affected by language barriers, as well as educational, cultural, social, and economic factors. Risk communicators must contend with the inherent uncertainty of many scientific findings, as well as the dissemination of misinformation in news and social media ${ }^{2}$. Federal government agencies play a prominent role in designing and disseminating risk messages despite these complexities. The objectives of these agencies go beyond simple education and information sharing; agencies must build public trust and achieve risk-mitigating behaviour change at a national level. As novel and complex problems arise, new needs emerge (Table 1 ).

\footnotetext{
Applying risk communication science Agencies involved in risk communication efforts tend to implement-to the extent feasible-research from multiple disciplines, including science communication and the decision and risk management sciences. For example, the US National Oceanic and Atmospheric Administration (NOAA) emphasizes the importance of having an informed plan, speaking to the interests of (and testing messages with) the intended audience, explaining risks with stories and visuals, offering options for reducing risk, working with trusted sources and the public, and using multiple communication methods ${ }^{3}$. Although in many cases the
}

extant literature is actionable, agencies often struggle to translate the best evidence to their specific needs. For example, despite guidance from researchers to present absolute risk (for example, "this drug will lead to side effects in 10 out of 1,000 patients who take it") rather than other risk formats ${ }^{4}$, the US Food and Drug Administration (FDA) cannot do so because it usually has access only to reported cases and not the associated base rates necessary to calculate absolute risk. Moreover, the FDA needs to communicate about new and emerging safety risks associated with prescription and over-the-counter medications and has found that research-guided recommendations rarely include guidance on communicating uncertainties. Several agencies have observed that the existing literature focuses more closely on communicating harms than on communicating uncertainty or even communicating benefits (for example, of a new technology or medication that balances risk $)^{5}$. Some relevant work suggests that people have clearly different responses to unique sources of scientific uncertainty ${ }^{6}$, highlighting the importance of tailoring messages to fit the specific type of uncertainty and targeted population.

A perpetual challenge faced by US agencies is that effective strategies in some population subgroups are largely ineffective in others. For example, the US Environmental Protection Agency (EPA) used risk communication principles derived from conventional health behaviour theories to build the Smoke Sense mobile phone app to reduce the health burden of wildfire smoke. They observed that user groups engaged with the app in different ways, based on individual health conditions and other factors ${ }^{7}$. Variability in effectiveness across populations illustrates the critical importance of involving intended recipients when developing risk communications. For example, the
Agency for Toxic Substances and Disease Registry (ATSDR) must continually adapt its communications strategy to meet the needs of different audiences facing unique environmental contamination issues (for example, perfluorinated and polyfluorinated compounds in drinking water). Adding to the challenge, ATSDR often must engage a community without fully knowing either the level of exposure the community has had or the adverse health effects of the contaminant. To understand the most effective strategy for reaching its specific audiences, ATSDR works directly with affected communities via town hall meetings and focus groups. ATSDR also learns from community assistance panels that serve as liaisons between the agency and exposed communities. Using these methods, the agency gathers evidence about the knowledge, attitudes, and beliefs of exposed communities, equipping the agency to create effective communications.

\section{Engaging at-risk communities}

The importance of engaging relevant populations has been particularly important for the Centers for Disease Control and Prevention (CDC) in managing outbreaks of Ebola virus disease. Outbreak response partners faced many challenges including extraordinary cultural and linguistic diversity and community threats, including safety amidst armed conflict and other chronic and infectious diseases, as well as basic needs for food and water. As a result, the CDC has found it important to actively engage members of the community, especially in situations where time is of the essence to control exposure, identify and offer vaccine to contacts, isolate the sick; treat patients safely, bury the dead in a safe and dignified manner, and gain community members' support for these actions before implementing them. Recommended public 
Table 1 | Risk communication challenges experienced by US federal agencies and recommendations

Prominent challenges for risk communicators in Recommendations federal agencies

- Working around the inherent uncertainty of many scientific findings.

- Contending with the circulation of misinformation and disinformation in news and social media, which require different strategies to address.

- Effective strategies in some population subgroups are largely ineffective in others. For example, strategies that work well in some parts of the world may not align with customs, beliefs, and practices in other places.

- Access and receptiveness to risk communications by the intended audience are greatly affected by economic status, language barriers, and educational, cultural, and social factors.

- A lack of direction on implementing best practices from risk communication science, suggesting a greater need to integrate lessons learned from implementation science.

- Clearance processes and political sensitivities can impede timely action.

- No focal point for risk communication across agencies that could bring together risk communication practitioners and researchers working in government agencies, which can result in competing risk messages.

- Not all US federal agencies have the capacity to conduct their own problembased research, due to budgetary and other limitations.

health actions, including communication research and strategies, that work well in some parts of the world may not align with local customs, beliefs, and practices in other parts. The CDC and their partners continue to develop ways to integrate community feedback and social science insights into outbreak responses to ensure good fit between recommended actions and local contexts.

Many federal agencies consider audience engagement to be a key tenet of their risk communication strategy. For example, the NOAA Impact-Based Decision Support Services program provides event-specific interpretive information to emergency managers and other core partners to address the many challenges associated with different types of high-impact weather. Additionally, NOAA and other agencies work collaboratively with relevant populations to obtain information essential to message design that may not have been considered by agency staff. For example, pets are not typically considered part of the EPA's mission to protect human health and the environment; however, failing to consider
- Clearly explain risk with stories and visuals, offer options for reducing risk, and tailor messages to fit the specific type of uncertainty and targeted population.

- Develop mutual trust and acknowledge cultural differences with target communities.

- Gather evidence about the knowledge, attitudes, and beliefs of target communities so that messaging aligns with their world view.

- Construe risk communication as a dynamic and nonlinear process in which the target population is involved in multiple stages, so communication materials are effectively tailored.

- Integrate community feedback and social science insights into outbreak responses to ensure a good match between recommended actions and local contexts.

- Prioritize interaction and collaboration with scholars in implementation science and the science of science communication to better implement risk communication best practices.

- Balance the urgency of addressing time-sensitive hazards such as pandemics and weather events when applying risk communication literature.

- Agencies should foster collaboration, as it can improve sharing of evidence about the effectiveness of risk communication strategies in various contexts; reduce redundant, competing, and contradictory risk messages; and leverage staff and budget resources to better implement risk communication science.

their needs turned out to be a crucial barrier to reducing the impact of wildfire smoke. Research suggesting that the perceived danger for family pets affects evacuation decisions led the EPA to partner with other agencies and associations to develop risk communication materials specifically about domestic pets and large animals ${ }^{8}$. The US Geological Survey Cascades Volcano Observatory worked collaboratively with partners and stakeholders to develop complementary messages and products on volcano hazards and engaged in ongoing discussions with policymakers to ensure that risk information was appropriately tailored ${ }^{9}$. The Cascades Volcano Observatory's most popular information product was developed in response to and in collaboration with local educators. The US National Science Foundation (NSF)-supported Societal Experts Action Network is leveraging stakeholder involvement to produce essential and usable information about how to better communicate pandemic-related information (https:// www.nationalacademies.org/our-work/ societal-experts-action-network).

\section{Limits to implementation}

At the root of many agency struggles is a relative lack of direction on implementing risk communication best practices. All agencies seek to leverage foundational insights, such as the importance of framing risks appropriately, using plain language, reducing communication barriers, and clearly identifying actionable steps for risk mitigation. Yet translating these concepts into practice introduces additional barriers that need further empirical attention. This should not be surprising, given that process issues fall more centrally within the practice of community-based participatory research and the field of implementation science, which considers optimal processes for translating and implementing the findings of basic science in field settings ${ }^{10}$. A key lesson here is that agencies would benefit from greater interaction between scholars in the fields of risk communication and implementation science.

Federal agency attempts to apply the risk communication literature can advance knowledge by identifying the limitations of existing models, revealing boundary conditions, and illuminating gaps in the current science. The risk communication literature has concentrated disproportionally on health and medical applications, and samples in many studies are not necessarily representative of the populations with which agencies most directly interact. Moreover, the application of some principles is often impractical given extenuating circumstances such as a need to make very quick decisions and constraints inherent in message testing. Agencies engaged in risk communication efforts need to develop mutual trust, acknowledge cultural differences, tailor messages to specific populations, construe risk communication as a dynamic and nonlinear process in which the target population is involved in multiple stages, and balance the urgency of addressing time-sensitive hazards such as pandemics and weather events with these goals. In doing so, they must avoid exacerbating beliefs in misinformation, which can be a paradoxical outcome of attempting to correct those beliefs ${ }^{2}$.

US federal agencies face additional structural limitations that can hinder attempts at impactful risk communication. Clearance processes and political sensitivities can impede timely action. There is no formal venue for bringing together risk communication practitioners and researchers working in government agencies, which can result in competing risk messages. Budget limitations are also an important factor. Some agencies have been able to address gaps in the science by conducting 
their own problem-based researchfor example, the FDA collects data to understand how to increase comprehension, decrease risk perceptions, and attenuate unintended consequences, including medication discontinuation-but not all agencies have the capacity to do so. Notably, the FDA collaborates with the US National Institutes of Health (NIH) to fund research on tobacco control that directly informs their efforts to regulate tobacco products, a key example of how agencies can work synergistically to link research investments and practice to accelerate progress.

\section{Supporting relevant research}

As a counterpoint to these challenges, the US Government plays a significant role in supporting risk communication research. The NSF has long funded this type of research in several programs and recently repositioned its Science of Science program to focus on communication research more explicitly (https://www. nsf.gov/funding/pgm_summ.jsp?pims_ $\mathrm{id}=505730$ ). The NSF also emphasizes practical implications of the work it funds (defined as 'broader impacts'). At the NIH, risk communication research is typically integrated as part of health risk screening and risk reduction intervention research. During the SARS-CoV-2 pandemic, risk communication is playing a significant role in various NIH-supported research efforts, including initiatives to encourage testing in underserved communities (https://www.nih.gov/research-training/ medical-research-initiatives/radx/ radx-programs\#radx-up). An NIH team developed a primer to inform agencies involved in reducing vaccine hesitancy during the pandemic (https://obssr. od.nih.gov/wp-content/uploads/2020/12/ COVIDReport_Final.pdf). All the US agencies represented in this article and many others not represented have active intramural research programs that study risk communication needs relevant to their missions.

Successful attempts to inform the US public about wildfire smoke, polyfluorinated compounds in water, tornadoes, infectious diseases, and drug side effects have all been predicated on existing knowledge about how people process risk information. Although we can harness what we have learned to address many national public health issues, nontrivial gaps remain. The work of a cross-agency group of behavioural and decision scientists at US federal agencies that led to this commentary represents an example of the kind of collaboration that may be needed to this end. This group has focused on many trans-agency issues, including the nature of graduate education in the behavioural and social sciences, common needs in language and communication research, and interests in big data. Collaboration can improve sharing of evidence about the effectiveness of risk communication strategies in various contexts; reduce redundant, competing, and contradictory risk messages; and leverage staff and budget resources to improve implementation of risk communication science.

Such an effort can be hastened by integrating lessons from implementation science and the science of science communication. Implementation science also highlights the importance of stakeholder involvement in determining solutions and risk messaging about those solutions, as well as the need to tailor messages based on a wide variety of personal, cultural, and geographical differences ${ }^{10}$. Although we have focused here on US agencies, government organizations around the world also draw on the extant risk communication literature and thus stand to benefit as well. Stronger risk communication helps governments achieve their missions to protect lives and improve myriad outcomes population-wide. Strengthening the public endorsement of science and building capacity for science to provide even more returns constitute important co-benefits that will accrue along the way and will continue to provide benefits well into the future.

\section{William M. P. Klein (D) ${ }^{\square}$, Alycia K. Boutté (D), Heather Brake ${ }^{2}$, Madeline Beal ${ }^{3}$,} Katherine Lyon-Daniel ${ }^{4}$, Emily Eisenhauer (D), Monica Grasso6 , Bryan Hubbell (D), Karen E. Jenni ${ }^{8}{ }^{8}$, Christopher J. Lauer ${ }^{9}$, Arthur W. Lupia ${ }^{10,11}$, Christine E. Prue ${ }^{12}$, Paula Rausch ${ }^{13}$, Carl D. Shapiro (DD) 14 Michael D. Smith (D) ${ }^{15}$ and William T. Riley (D) 16 ${ }^{1}$ Division of Cancer Control and Population Sciences, National Cancer Institute, National Institutes of Health, Rockville, MD, USA. ${ }^{2}$ National Center for Environmental Health/Agency for Toxic Substances and Disease Registry, Centers for Disease Control and Prevention, Atlanta, GA, USA. ${ }^{3}$ US Environmental Protection Agency, Office of the Administrator, Washington, D.C., USA. ${ }^{4}$ Centers for Disease Control and Prevention, Atlanta, GA, USA. ${ }^{5}$ US Environmental Protection Agency, Office of Research and Development, Washington, D.C., USA. ${ }^{6}$ National Oceanic and Atmospheric
Administration, Silver Spring, MD, USA. ${ }^{7}$ Office of Research and Development, US Environmental Protection Agency, Research Triangle Park, NC, USA. ${ }^{8}$ US Geological Survey, Energy and Minerals Mission Area, Denver, CO, USA. ${ }^{9}$ Performance, Risk and Social Science Office, National Oceanic and Atmospheric Administration, Silver Spring, MD, USA. ${ }^{10}$ National Science Foundation, Alexandria, VA, USA. ${ }^{11}$ University of Michigan, Ann Arbor, MI, USA. ${ }^{12}$ National Center for Emerging and Zoonotic Infectious Diseases, Centers for Disease Control and Prevention, Atlanta, GA, USA. ${ }^{13}$ Center for Drug Evaluation and Research Office of Communications, US Food and Drug Administration, Silver Spring, MD, USA. ${ }^{14}$ US Geological Survey, Science and Decisions Center, Reston, VA, USA. ${ }^{15}$ Performance, Risk and Social Science Office, National Oceanic and Atmospheric Administration, Silver Spring, MD, USA. ${ }^{16}$ Office of Behavioral and Social Sciences Research, National Institutes of Health, Bethesda, MD, USA.

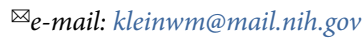

Published online: 18 March 2021

https://doi.org/10.1038/s41562-021-01081-0

References

1. Klein, W. M. P., Ferrer, R. A. \& Kaufman, A. R. JAMA Oncol. 6, 983-984 (2020).

2. Lewandowsky, S., Ecker, U. K. H., Seifert, C. M., Schwarz, N. \& Cook, J. Psychol. Sci. Public Interest 13, 106-131 (2012).

3. NOAA Social Science Committee. Risk Communication and Behavior: Best Practices and Research Findings. Technical Report https://www.performance.noaa.gov/wp-content/uploads/ Risk-Communication-and-Behavior-Best-Practices-andResearch-Findings-July-2016.pdf (National Oceanic and Atmospheric Administration, 2016).

4. Berry, D. C., Knapp, P. \& Raynor, T. Patient Educ. Couns. 63, 89-96 (2006).

5. Savadori, L. et al. Risk Anal. 24, 1289-1299 (2004).

6. Han, P. K. J. et al. Patient Educ. Couns. 102, 1756-1766 (2019).

7. Rappold, A. G. et al. Geohealth 3, 443-457 (2019).

8. US Environmental Protection Agency. Protect your pets from wildfire smoke factsheet. AirNow.gov https:// www.airnow.gov/publications/wildfire-smoke-guide/ wildfire-smoke-protect-your-pets/ (2019).

9. Ludwig, K.A. et al. Science for a risky world-A U.S. Geological Survey plan for risk research and applications Circular 1444 https://doi.org/10.3133/cir1444 (U.S. Geological Survey, 2018).

10. Peters, D. H., Adam, T., Alonge, O., Agyepong, I. A. \& Tran, N. Br. Med. J. 347, f6753 (2013).

\section{Acknowledgements}

There are no relevant funding sources to report. This article has been internally peer reviewed and approved for publication consistent with US Geological Survey Fundamental Science Practices.

\section{Competing interests}

All authors are employed by US federal agencies. Views expressed in this article are of the individual authors and do not necessarily reflect the views or policies of the NIH, EPA, NOAA, NSF, FDA, or CDC.

\section{Additional information}

Peer review information Nature Human Behaviour thanks Dominique Brossard for their contribution to the peer review of this work. 